\title{
MobileQuiz: A Serious Game for Enhancing the Physical and Cognitive Abilities of Older Adults
}

\author{
Thomas Birn, Clemens Holzmann, and Walter Stech \\ Universitiy of Applied Sciences Upper Austria \\ Department of Mobile Computing \\ Softwarepark 11, 4232 Hagenberg, Austria \\ http://www.fh-hagenberg. at
}

\begin{abstract}
The ageing process involves physical and cognitive challenges. It is a known fact that (outdoor) physical activity can help to counter these issues and improve the quality of life. One way to motivate older adults doing exercises are serious games. They embody the concept of game-based learning and exercising, and they are designed to solve a problem along with providing and engaging training experience. Based on recent research, we have developed a concept of an outdoor serious game, which has been designed to keep older adults mobile and enhance their cognitive abilities at the same time. We have developed a prototype and evaluated it in a user study with elderly participants. The results show a high acceptance by the test participants, indicating that this kind of game is interesting for the target group. The usability of the prototype has also been evaluated and shows good average scores.
\end{abstract}

Keywords: Serious games, accessible games, design for aging.

\section{Introduction}

The great increase of the old population and the increasing social costs are urgent issues society should address with proper plans [1]. By 2060, the US population of adults over 65 is expected to increase from 43 to 92 million [2]. This increase has direct and severe implications for society and individuals as well. It is therefore in the society's best interest to find solutions that will keep people healthy and mobile at low cost. With age come certain changes to the human body resulting in various difficulties and challenges. These can be either of physical nature (affecting hearing, vision and motor skills) or of mental nature (including difficulties in perception, attention and memory) [3]. It is a known fact that next to purely mental training, frequent physical activity can help to counter not only the physical challenges, but also positively influences the mental apparatus [4]. Investigations showed, that especially outdoor physical activity has potential to improve the mental health $[5,6]$. Based on recent research, we conclude that regular, combined physical and mental training has the potential to improve the quality of life of older adults.

An important success factor in getting elderly to perform exercises on a regular basis is their motivation. This motivation can be increased by the help of

C. Stephanidis and M. Antona (Eds.): UAHCI/HCII 2014, Part III, LNCS 8515, pp. 3-14, 2014.

(C) Springer International Publishing Switzerland 2014 
serious games, which offer concepts for motivating their players. They embody game-based learning and exercising, and they are designed to solve a problem along with providing and engaging training experience [7]. In contrast to games for pure entertainment, the focus of serious games lies on the training aspect. A higher motivation can be achieved by using a set of well known gratification and motivation techniques, like for example a scoring system [8] or the implementation of a social component, as suggested by Planic et al. [8] and Brox et al. [9].

The goal of the presented work was to develop a location-based, serious game for elderly people, which combines physical and mental training in a way that keeps them motivated to play on a regular basis. From a technical viewpoint, the ideal computing platform for this purpose are state-of-the-art smartphones, which are equipped with a sophisticated set of sensors and considerable processing power. In particular, features such as included GPS, digital compass and inertial sensors as well as a big screens make them attractive for mobile locationbased games. Moreover, a representative survey targeting the elderly of five EU countries has concluded that the age-divide for mobile phone usage is narrowing faster than for regular computer use [10]. However, although more and more older adults are familiar with smartphones, existing interface designs may not be suitable for the majority of them [11]. This requires a special consideration of universal access in the design phase of mobile applications for the elderly.

In this paper, we propose a serious game called MobileQuiz that combines the above mentioned aspects: The physical and mental training in a game context that is both accessible and usable for older adults, and which will motivate them to play. In the following section we will present the related concepts. We will then proceed with a detailed description of our game concept, the technical approach and a prototypical implementation for Android phones. Afterwards, we will present a first evaluation of the prototype which has been carried out with 8 participants.

\section{Related Work}

Recent research showed a huge number of game concepts designed to foster the physical strength of its players. Kyung-Sik et al. [12] studied a game design in which the player is prompted to control an avatar with a foot board and hand-held controllers. The study "Exergames for Elderly" investigated the use of the Nintendo Wii Fit ${ }^{1}$ platform to enhance physical fitness [9]. Burke et al. investigated a game, in which the player has to catch oranges with a physical basket [13]. Games, that are played with mobile devices for enhancing the physical strength are e.g. "Penguin Toss" and "Bowling" as developed by Sunwoo et al. [14]. In contrast to our concept however, these games are not optimized for older adults and their key purpose is limited to physical training.

A serious game for enhancing the mental fitness is "ElderGames", a memorylike game played on a table [15]. Another example is the serious game described

${ }^{1}$ http://wiifit.com/ 
in [16], which should stimulate the cognitive abilities of Altzheimer's patients by letting them resemble daily life activities in a game. These games are designed for older adults with the aim to improve their cognitive abilities, physical training aspect are not included though.

Besides stationary games as mentioned above, recent work investigated location-based games that are played outdoors and on mobile devices. In the mobile fitness game "SmartRabbit" for example, the goal is to run a certain distance in the shortest time possible [17]. Another example is the "Business Consultant" game [18], which guides the player through a set of nearby locations, who has to conduct virtual interviews at each target location. The difference of such games to our concept lies in the lack of optimization for older adults.

As summarized in Table 1, the majority of these works make use of one single training aspect or focus on the technical game development, but they lack an optimization for older adults. Because little has been done to address the issue of digital game design for older adults with the focus on physical exertion together with mental training [3], our approach combines those two concepts with a focus on user experience for older adults and the motivation aspect.

Table 1. A summary of the training and game aspects of the related concepts

\begin{tabular}{|l||c|c|c|c|c|c|c|c|}
\hline & {$[9]$} & {$[12]$} & {$[13]$} & {$[14]$} & {$[15]$} & {$[16]$} & {$[17]$} & {$[18]$} \\
\hline \hline Designed for seniors & & & & & $\otimes$ & $\otimes$ & & \\
\hline Physical training & $\otimes$ & $\otimes$ & $\otimes$ & $\otimes$ & & & $\otimes$ & $\otimes$ \\
\hline Mental training & & & & & $\otimes$ & $\otimes$ & & $\otimes$ \\
\hline Mobile device & & & & $\otimes$ & & & $\otimes$ & $\otimes$ \\
\hline
\end{tabular}

\section{MobileQuiz Concept and Characteristics}

MobileQuiz is a serious game where the player has to conduct an outdoor challenge based on the principles of geocaching. The goal is to find and reach predefined locations based on spatial cues. Once a location has been reached, the player has to answer questions in order to get points and unlock the information about how to reach the next location. The game design aims to (i) be elder friendly, (ii) enhance the player's physical mobility, (iii) strengthen the player's mental ability and (iv) orientation, while (v) motivating him or her to play the game again. In order to achieve those aims, the concept includes different components that cover the respective areas as shown in Table 2 and explained in the following concept description.

The key aspect of the game is the wayfinding (WF) the users have to perform while playing the game. It combines the physical workout when walking from destination to destination with mental training when looking for the right way 
Table 2. The overall aims are mapped to the concept components: Wayfinding (WF), Map (MA), Quiz (QZ), User Interface (UI) and Personalization (PE)

\begin{tabular}{|l||c|c|c|c|c|}
\hline & WF & MA & QZ & UI & PE \\
\hline \hline (i) Senior friendly & & & & $\otimes$ & \\
\hline (ii) Physical fitness & $\otimes$ & & & & \\
\hline (iii) Mental fitness & $\otimes$ & & $\otimes$ & & \\
\hline (iv) Orientation training & $\otimes$ & $\otimes$ & & & \\
\hline (v) Motivation & & & $\otimes$ & & $\otimes$ \\
\hline
\end{tabular}

to go. The wayfinding forces the players to orientate themselves. A map (MA) visualization and directions also add up to the orientation training aspect. The gaming component of the concept - the quiz - will train the mental fitness of the players while giving additional motivation to them when receiving points for right answers (QZ). A specifically adapted user interface makes sure that the game suits the needs of older adults when it comes to the interface design. The game can be personalized (PE) in terms of two game modes. This ensures additional motivation as the game has a wider application.

The game is entirely played outdoors. At the beginning of each game, the player can choose between two different game modes. Both will provide different settings and advantages as described in Section 3.1. However, the basic game principles stay the same. After the selection of a mode, the current game setting includes a set of at least two different locations the player has to visit successively by foot. An analog compass, together with the distance to the next location (see Figure 1a), is shown at the display of the mobile device and helps the player to reach the destination. If the player is not able to find the location, a navigation function is included, which shows the shortest path to the destination on a map and gives turn-by-turn directions if desired. How locations are added to the game will be explained in more detail in the following sections.

The access to the game questions is triggered by the GPS module of the mobile device whenever the next destination has been reached. At this point, the player has to answer questions that are either related to the location or to the region surrounding that location. A screenshot with an example question is shown in Figure 1b. The player gets points once a destination has been reached and when answering a question correctly. Once all the questions for the location are answered, the compass that shows the direction to the next location is unlocked and shown on the display. This cycle repeats until all locations have been visited. At the end of the game, a summary of the finished game is shown to the player. Since all game runs are stored on the device, the player can revise the played games at any time including a map of the actual path the player travelled and the list of questions that have been answered. The correct and chosen answers are highlighted in different colors. 


\subsection{Game Modes}

The proposed game has two modes, an automatic and a manual one:

- Automatic Mode: The automatic mode uses nearby points of interest to generate a game run. The player has the option to specify the minimum and maximum number of locations to be added to the game, which allows for an approximate estimation of the game duration.

- Manual Mode: In this mode, the player is able to use custom locations in the game. A search function provides a category-based search of nearby points of interest. After a successful search, certain points of interest can be added as new location to the game. Previously found and selected locations can always be re-selected for future game runs.

Providing a manual in addition to an automatic game modes is mainly for two reasons. First, the game can be better personalized to the needs of the player by supporting custom locations. Second, it provides the opportunity to alter the game in a way that allows for a better integration into everyday life, which in turn adds to the motivation aspect. This combination of modes allows for different usage scenarios, ranging from the integration of the game with simple daily routines to the exploration of unknown cities.

Each mode will guide the player back to the starting location when the last location of a game has been reached. This allows the user to get back to the starting point, which is assumed to be a well known location. Otherwise, the player may find himself in an unfamiliar area once the game has been finishes.

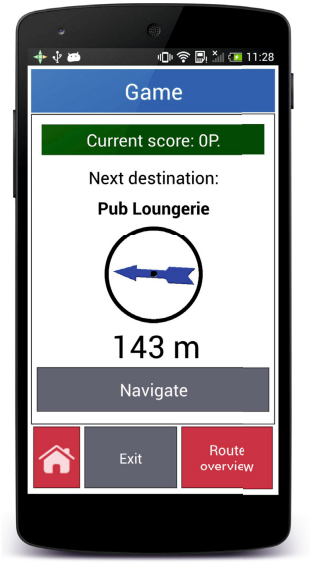

(a) Game screen

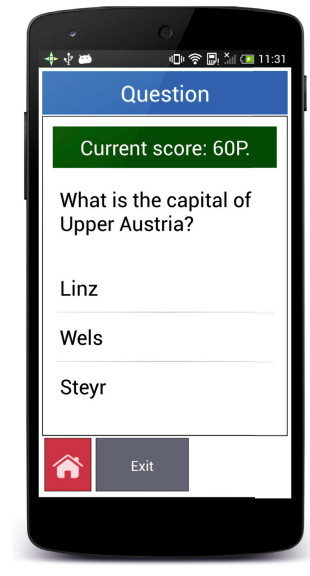

(b) Question screen

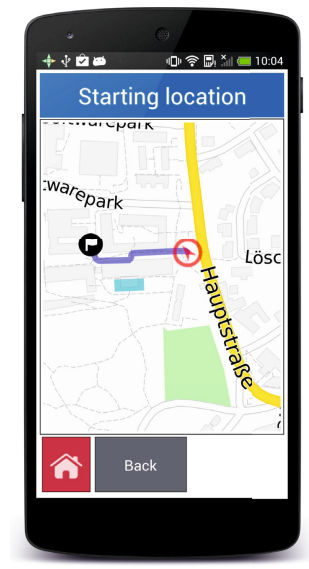

(c) Map screen

Fig. 1. The game screen (a) shows the direction and distance to the next destination. After the destination is reached, the player receives points and has to answer a question (b). A map screen (c) provides guidance to the next destination when the "navigate" button is pressed. 


\subsection{User Interface Design}

The user interface of the game has been designed according to several guidelines for graphical building user interfaces for older adults $[5,8,11]$. It has been kept simple while showing task-relevant information only. The use of big fonts and a high contrast color scheme additionally adds to the accessibility for older adults. The basic layout of the game stays the same for all views. In order to be more consistent throughout the service, the layout of all screens includes buttons with a positive-negative-semantic. The left ("negative") button will always undo the last action or exit the current state. The right ("positive") button will trigger the most reasonable action (for example: saving a location, starting a game, etc.); it is disabled if no action is available. The design is depicted in Figure 1.

\section{Technical Approach}

A fully functional prototype implementation of the service has been built following the described concept. One major challenge was to retrieve nearby locations as well as questions that are related to these locations. Our approach to generate a quiz route includes three main steps that are shown in Figure 2. At first, the searching and processing of nearby points of interest is needed. As we are not aware of any existing toolkits or public APIs for generating location-based questions, this is a key aspect of the technical implementation we had to face. The third step is the combination of generated locations and questions to a reasonable quiz route.

3) Generate route

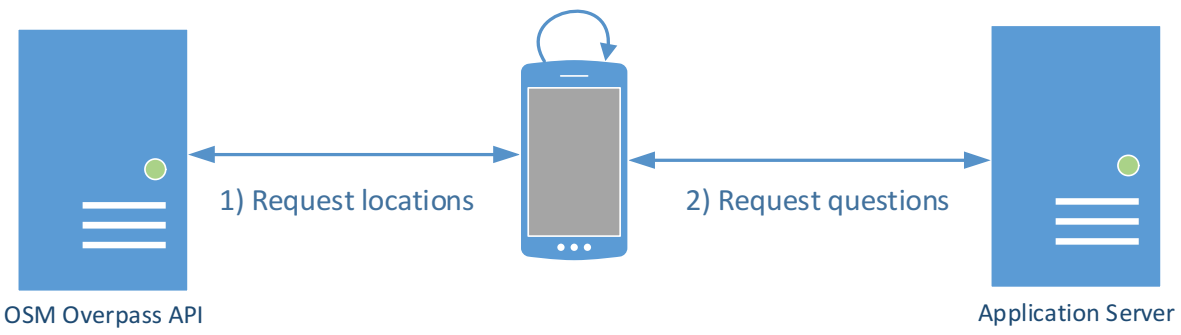

Fig. 2. Generating a quiz route includes three steps

\subsection{Requesting Nearby Locations}

The first step of requesting nearby locations is only necessary if the player chooses the automatic mode, since the manual mode already includes self-defined locations. Once the player hits the "Start Game" button, the device will conduct a search for nearby locations. For the prototype implementation, the surrounding search diameter has been limited to 800 meters, which has shown to be a 
reasonable distance in initial tests. In order to get publicly available points of interest, we used the Overpass API of the OpenStreetMap ${ }^{2}$ (OSM) project. The API request can be parameterized with the following metrics:

- The center of the search that will be conducted,

- the diameter of the search area, and

- the types of points of interest.

The result is a list of nearby points of interest fitting the parameters. The types of the requested points of interest were limited to a subset of most reasonable types. Instead of including arbitrary location types like toilets, we focused on noteworthy places like common sights, places of worship and parks, but also supermarkets, restaurants and banks.

\subsection{Requesting Quiz Questions}

The automatic generation of location-based quiz questions is a complex task and would require huge efforts to implement. For the prototype implementation, the quiz questions were therefore provided manually via a web interface on the application server. A question can be defined by setting the following parameters:

- The coordinates of its center,

- a radius defining the validity of the question,

- the question itself,

- and a set of answer possibilities where the correct one is marked.

The specified radius defines a diameter around the center of the question, defining its area of validity. This allows for a generic and flexible way to create questions as they can be set to be valid for a relatively small area (for example a building or a park) or for wide areas (like country regions or even whole districts). It is important to note, that this approach could be improved by adding the option to use polygons for defining areas. This would allow for a more detailed level of definition.

\subsection{Generating a Quiz Route}

Generating a quiz route on the mobile device includes the previously mentioned fetching of locations and retrieving of quiz questions from the application server. The locations and questions are then merged to a game route. The process consists of the following steps:

1. Fetching of surrounding points of interest from OSM data for the automatic mode or fetching all surrounding custom locations in manual mode.

2. Calculating the diameter that is formed by all fetched nearby locations.

3. This diameter and the user's location are sent to the application server in order to request suitable questions. The server returns all questions whose diameters overlap with that of the nearby locations.

2 http://www .openstreetmap.org 
4. The fetched points of interest and questions are then merged on the mobile device by assigning each question the closest point of interest (if it has not been assigned to another question yet). This is done under the assumption that spatial proximity is a good indicator for the relation between a certain question and a geographic location.

5. The last step in the process uses methods of heuristic optimization to calculate the "best" route that includes all selected locations. We define the best route as a connection between all points of interest so that no cycles and no double visits of sub-paths occur. For the route generation, just points of interest which have been assigned to a question are considered. For the prototype implementation, we solved this travelling salesman problem with the techniques of simulated annealing, what provides good results in a reasonable time for our prototype implementation. Because of already stated reasons, the starting location is added to the game as the fixed destination before the calculation begins.

Once the game route has been generated, the player can navigate to the first location.

\section{Evaluation}

The prototype has been evaluated with user tests. The main purpose was to assess the usability and acceptance of the concept in an initial study. The questions of interest are whether such a game is accepted by the target group or not, and if the provided concept is sufficiently usable. An assessment of specific physical and mental improvements has not been made. The evaluation of this would need long-term studies in an controlled environment. Therefore, the focus was on

1. the assessment of the usability of the concept and the user interface,

2. the observation how intuitive the prototype was to use for the participants,

3. the identification of potential design flaws, and

4. the assessment if this kind of game will be accepted by the target group.

\subsection{Methodology and Setup}

The tests were performed by eight participants, 3 male and 5 female, between the age of 60 and $71(\mathrm{M}=65.8, \mathrm{SD}=3.9)$. The small sample size should be sufficient to gain insights into the most common usability problems [19] and to get an estimate about the user satisfaction. Four of the participants are using a feature phone regularly for making telephone calls. Two of the participants had already used a smart phone before, the rest had not have used either a smart phone or a feature phone before. The participants had to perform tasks using the prototype, which included one actual game run and some tasks to configure the game as well as reviewing played games. Overall, there were four different assignments: 
1. Start an automatic game and play it to the end.

2. Add a place to a manual game.

3. View the location properties of an already defined location.

4. Review the previously played game and look at your answers to the questions.

For the test run, we created a set of questions in the surrounding areas of the test participants via the web interface. The questions were related to the area and some nearby points of interest. During the execution of the tasks, we gathered the times it took to complete them as well as data from observations by the study instructor. We also advised the participants to think aloud during the tests. The sequence of the tasks had to be performed three times in order to assess the learning curve of the game. After finishing all scenarios, the participants were asked to fill out the System Usability Scale (SUS) [21] questionnaire to assess the overall system usability.

\subsection{Timing Results}

All participants were able to finish all tasks. The first task included the navigation from one location to another in different settings. To compensate for the resulting differences in task times, only the duration for starting the application and for configuring and starting the game has been taken into account for task one. Figure 3a shows how much time it took the participants in average to perform the task sequence for each run. The lowest time for the first run was 213 seconds for the slowest participant and 196 seconds for the fastest with a mean of 216.13 ( $\mathrm{SD}=15.71$ ) seconds. The second run was faster with the slowest time of 168 seconds, the fastest time of 154 seconds and a mean of 159.63 seconds $(\mathrm{SD}=4.98)$. In the third run, the slowest time was 161 seconds, the fastest time was 141 seconds and the mean time was 151.38 seconds $(\mathrm{SD}=6.99)$.

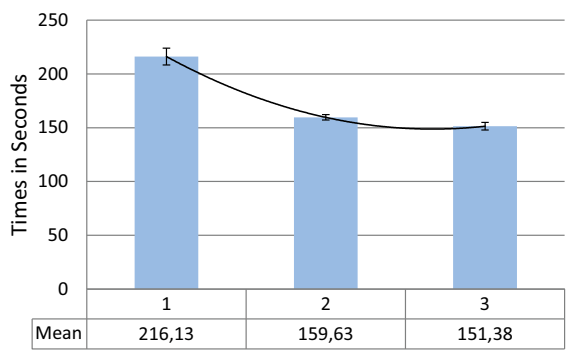

(a) Times per trial

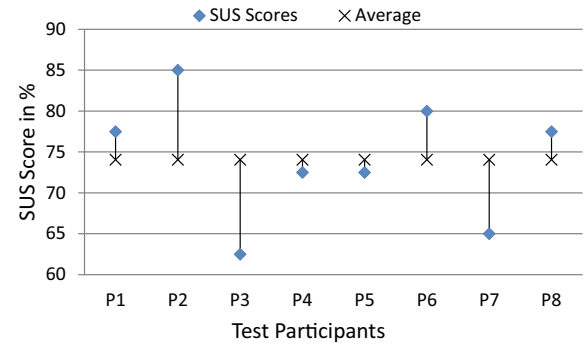

(b) SUS score per participant

Fig. 3. The mean task times (a) show an improvement of $30 \%$ for the third run compared to the first run. The SUS scores per participants (b) show an overall good usability. 


\subsection{User Feedback}

After finishing all test scenarios, the participants were asked to give feedback and some ideas of potential design flaws and potential for improvement. Some participants mentioned the usefulness of an additional audio feedback when approaching a destination. This way, the visual focus would not lie on the display of the mobile device all the time. During the execution of task two, some participants had a hard time when picking a point of interest from the map. They claimed that the zoom level is too low. In general, the participants had troubles when interacting with the map. When asked for their opinion about the tasks, some participants stressed that they would prefer a simpler user interface for task two, the adding of a new location to the manual game. The remaining tasks were perceived as positive and usable by the participants. When asked if the participants had fun playing the game, all but one of them totally agreed. All but one participants would also recommend the game to friends. Additionally, the participants were asked to fill out the SUS questionnaire; the aggregated result shows an overall SUS score of $75(\mathrm{SD}=7.55)$ on a scale from 1 to 100. Figure $3 \mathrm{~b}$ shows the SUS score per participant with the lowest value of 63 and a highest value of 85 .

\subsection{Discussion}

The results of the eight individual test runs can be seen as outcome of an initial study and are not significant due to the small sample size. They allow, however, the drawing of conclusions concerning the user satisfaction of the evaluated game and the overall experience of such kind of games. The gathered quantitative data show a clear learning curve with a mean improvement of $30 \%$ $(\min =23.3 \%, \max =34.1 \%)$ for the third trial compared to the first run (see Fig. 3a). This indicates that the participants, which had little to non experience with smart phones, are able to learn the used procedures in the game when playing regularly. The mean SUS score of 75 shows the general good usability of the system. Some participants had troubles with the map interaction. We observed that the participants could not handle the necessary multi touch gestures very well. We also did not not provide any alternative means of interacting with the map like for example buttons for zooming. Additionally, the zoom level has been perceived as too low. The study revealed the need of two major improvements of the system: (a) the auditory feedback of game states and (b) the improvement of the map in terms of interaction and zoom levels. The user feedback clearly shows that the game has been widely accepted by the test participants. This indicates that this type of game can be fun to play and would motivate older adults to do exercising.

\section{Conclusion and Future Work}

The introduced concept combines physical exertion and cognitive training to one outdoor game. The design of the concept defines different components in order to 
reach the concept aims. A prototype has been implemented following a profound technical approach and evaluated in a user study. The main purpose of the study was to show how this kind of games will be accepted by the target group and if the presented concept implementation is usable. We showed that the majority of the test participants are satisfied with the usefulness of the concept and the provided usability. The participants had fun playing the game and the majority would recommend it to friends. We see potential for future investigation in the visualization and interaction of maps in mobile, location-based serious games, as well as in the automatic generation of context based quiz questions. Another future development could be the introduction of a social component to share game results in order to motivate the player further, as suggested by Planic et al. [8] and Brox et al. [9].

Acknowledgement. The MobileQuiz game has been developed as a part of the MOBILE.OLD project. The project MOBILE.OLD acknowledges the financial support within the Ambient Assisted Living (AAL) programme funded by the European Union and the respective "National Research Promotion Programmes" of the federal ministries of the partner countries Austria, Germany, Spain, Romania, UK and The Netherlands.

\section{References}

1. Nehmer, J., Becker, M., Karshmer, A., Lamm, R.: Living assistance systems: an ambient intelligence approach. In: 28th International Conference on Software Engineering, pp. 43-50 (2006)

2. United States Census Bureau (2014), http://www.census.gov/ (accessed January 2014)

3. Gerling, K.M., Schild, J., Masuch, M.: Exergame design for elderly users: the case study of SilverBalance. In: 7th International Conference on Advances in Computer Entertainment, pp. 66-69 (2010)

4. Center for Desease Control and Prevention (2013), http://www.cdc.gov/physicalactivity/everyone/health/index.html (accessed September 2013)

5. Zajicek, M.: Interface Design for Older Adults. In: EC/NSF Workshop on Universal Accessibility of Ubiquitous Computing, pp. 60-65 (2001)

6. Pretty, J., Peacock, J., Sellens, M., Griften, M.: The mental and physical health outcomes of green exercise. International Journal of Environmental Health Research 15(5), 319-337 (2005)

7. Tan, C.T., Soh, D.: Augmented Reality Games: A Review. In: Proc. GAMEONARABI. European Multidiscilinary Society for Modelling and Simulation Technology (2010)

8. Planic, R., Isabella, N., Kampel, M.: Exergame Design Guidelines for Enhancing Elderly's Physical and Social Activities. In: 3rd International Conference on Ambient Computing, Applications, Services and Technologies, pp. 58-63 (2013)

9. Brox, E., Luque, L.F., Evertsen, G.J., Hernandez, J.E.G.: Exergames for elderly: Social exergames to persuade seniors to increase physical activity. In: 5th International Conference on Pervasive Computing Technologies for Healthcare, pp. 546549 (2011) 
10. European Commission: Seniorwatch 2 - Assessment of the Senior Market for ICT Progress and Developments (2008), http://ec.europa.eu/information_society/newsroom/cf/ document.cfm?action=display\&doc_id=526 (accessed January 30, 2014)

11. Kurniawan, S.: Older people and mobile phones: A multi-method investigation. In: Human-Computer Studies, vol. 66, pp. 889-901 (2008)

12. Kyung-Sik, K., Seong-Suk, O., Jin-Ho, A., Sun-Hyung, L.: Development of a walking game for the elderly using controllers of hand buttons and foot boards. In: 17th International Conference on Computer Games, pp. 158-161 (2012)

13. Burke, J.W., McNeill, M.D.J., Charles, D.K., Morrow, P.J., Crosbie, J.H., McDonough, S.M.: Serious Games for Upper Limb Rehabilitation Following Stroke. In: Games and Virtual Worlds for Serious Applications, pp. 103-110 (2009)

14. Sunwoo, J., Wallace, Y., Lutteroth, C., Wünsche, B.: Mobile games for elderly healthcare. In: 11th International Conference of the NZ Chapter of the ACM Special Interest Group on Human-Computer Interaction, pp. 73-76 (2010)

15. Gamberini, L., Fabregat, M., Spagnolli, A., Prontu, L., Seragila, B., Alcaniz, M., Zimmermann, A., Rontti, T., Grant, J., Jensen, R., Gonzales, A.L.: Eldergames: Videogames for empowering, training and monitoring elderly cognitive capabilities. In: 6th International Conference of the International Society for Gerontechnology, vol. $7(2)$, p. $111(2008)$

16. Imbeault, F., Bouchard, B., Bouzouane, A.: Serious games in cognitive training for Alzheimers patients. In: 1st International Conference on Serious Games and Applications for Health, pp. 1-8 (2011)

17. Marins, D.R., de Justo, M.O.D., de Chavec, B.A.M., D'Ipolitto, C.: SmartRabbit: A Mobile Exergame Using Geolocation. In: 2011 Symposium on Games and Digital Entertainment, pp. 232-240 (2011)

18. Parsons, D., Petrova, K., Hokyoung, R.: Mobile Gaming - A Serious Business! In: 7th International Conference on Wireless, Mobile and Ubiquitous Technology in Education, pp. 17-24 (2012)

19. Nielsen, J.: Estimating the number of subjects needed for thinking aloud test. International Journal of Human-Computer Studies, 385-397 (1994)

20. Lewis, J.R.: IBM Computer Usability Satisfaction Questionnaires. Phsychometric Evaluation and Instructions for Use. International Journal of Human-Computer Interaction, 57-78 (1995)

21. Brooke, J.: SUS - A quick and dirty usability scale. Usablity Evaluation in Industry 1, 189-194 (1996) 